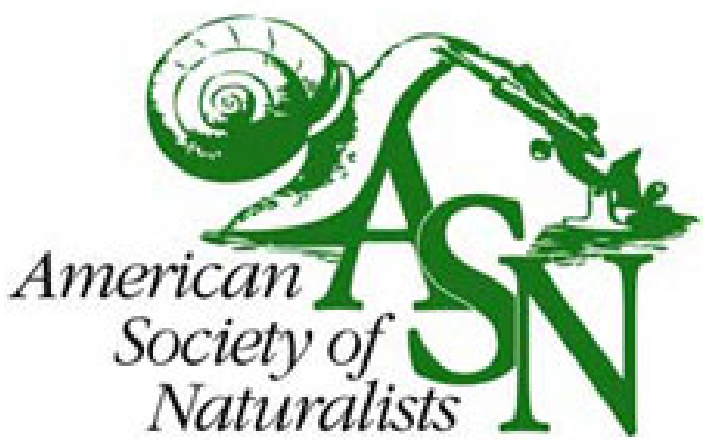

Distinguishing Social from Nonsocial Navigation in Moving Animal Groups. Author(s): Nikolai W. F. Bode, Daniel W. Franks, A. Jamie Wood, Julius J. B. Piercy, Darren P. Croft, Edward A. Codling

Reviewed work(s):

Source: The American Naturalist, Vol. 179, No. 5 (May 2012), pp. 621-632

Published by: The University of Chicago Press for The American Society of Naturalists Stable URL: http://www.jstor.org/stable/10.1086/665005

Accessed: 16/04/2012 10:04

Your use of the JSTOR archive indicates your acceptance of the Terms \& Conditions of Use, available at http://www.jstor.org/page/info/about/policies/terms.jsp

JSTOR is a not-for-profit service that helps scholars, researchers, and students discover, use, and build upon a wide range of content in a trusted digital archive. We use information technology and tools to increase productivity and facilitate new forms of scholarship. For more information about JSTOR, please contact support@ jstor.org. 


\title{
Distinguishing Social from Nonsocial Navigation in Moving Animal Groups
}

\author{
Nikolai W. F. Bode, ${ }^{1,2, \star}$ Daniel W. Franks, ${ }^{1,3}$ A. Jamie Wood, ${ }^{1,4}$ Julius J. B. Piercy, ${ }^{5}$ \\ Darren P. Croft, ${ }^{6}$ and Edward A. Codling ${ }^{5,7}$
}

1. York Centre for Complex Systems Analysis, Ron Cooke Hub, University of York, York YO10 5GE, United Kingdom; and Department of Biology, University of York, York YO10 5YW, United Kingdom; 2. Department of Ecology and Evolutionary Biology, Princeton University, Princeton, New Jersey 08544; 3. Department of Computer Science, University of York, York YO10 5DD, United Kingdom; 4. Department of Mathematics, University of York, York YO10 5DD, United Kingdom; 5. School of Biological Sciences, University of Essex, Colchester CO4 3SQ, United Kingdom; 6. Centre for Research in Animal Behaviour, College of Life and Environmental Sciences, University of Exeter, Exeter EX4 4QG, United Kingdom; 7. School of Mathematical Sciences, University of Essex, Colchester CO4 3SQ, United Kingdom

Submitted June 8, 2011; Accepted January 10, 2012; Electronically published March 28, 2012

Online enhancement: appendix. Dryad data: http://dx.doi.org/10.5061/dryad.kt3109v7.

ABSTRACT: Many animals, such as migrating shoals of fish, navigate in groups. Knowing the mechanisms involved in animal navigation is important when it comes to explaining navigation accuracy, dispersal patterns, population and evolutionary dynamics, and consequently, the design of conservation strategies. When navigating toward a common target, animals could interact socially by sharing available information directly or indirectly, or each individual could navigate by itself and aggregations may not disperse because all animals are moving toward the same target. Here we present an analysis technique that uses individual movement trajectories to determine the extent to which individuals in navigating groups interact socially, given knowledge of their target. The basic idea of our approach is that the movement directions of individuals arise from a combination of responses to the environment and to other individuals. We estimate the relative importance of these responses, distinguishing between social and nonsocial interactions. We develop and test our method, using simulated groups, and we demonstrate its applicability to empirical data in a case study on groups of guppies moving toward shelter in a tank. Our approach is generic and can be extended to different scenarios of animal group movement.

Keywords: animal behavior, animal movement, sociality, animal migration, group navigation, collective behavior.

\section{Introduction}

Many animals, such as migrating flocks of birds, schools of fish, or herds of ungulates, navigate in groups (Krause and Ruxton 2002). However, moving in a group does not necessarily imply that group members interact socially by sharing available information, either directly or indirectly.

* Corresponding author; e-mail: nbode@princeton.edu.

Am. Nat. 2012. Vol. 179, pp. 621-632. (c) 2012 by The University of Chicago. 0003-0147/2012/17905-53091\$15.00. All rights reserved. DOI: $10.1086 / 665005$
If each individual in an initially aggregated collection of animals was attracted to the same resource (e.g., food, females, shelter), then the aggregation could remain stable without social interactions, making it difficult to distinguish this scenario from a navigating social group (see fig. 1 for an illustration). For example, fish larvae settling on a reef (Codling et al. 2004 and references therein), migrating birds (Bajec and Heppner 2009), swarming locusts (Buhl et al. 2006), and possibly even humans walking toward similar targets (see Faria et al. 2009 for experiments) may interact with different levels of sociality. It has also been suggested that different levels of motivation (e.g., hunger) can significantly alter the outcome of group navigation (Conradt et al. 2009). Such variations in behavioral state could result in varying levels of sociality even between groups from a single species. Throughout this research we assumed that group formation has occurred and that we were observing a moving aggregation of animals. How often entirely nonsocial navigating groups exist is likely to depend on how often nonsocial aggregation occurs in the first place, but it is also possible that differences in the level of sociality in groups could lead to social aggregation followed by less social navigation.

For animals, the distinction between social and nonsocial navigation is likely to be important. For example, it has been suggested that social navigation could facilitate finding and/or learning of the most beneficial or least dangerous migratory route in juvenile birds (Mellone et al. 2011). Theoretical research has shown that in some circumstances asocial groups, in which individuals interact only to avoid collisions, achieve less efficient navigation than social groups, in which individuals actively maintain group cohesion and alignment (Codling et al. 2007). Fur- 

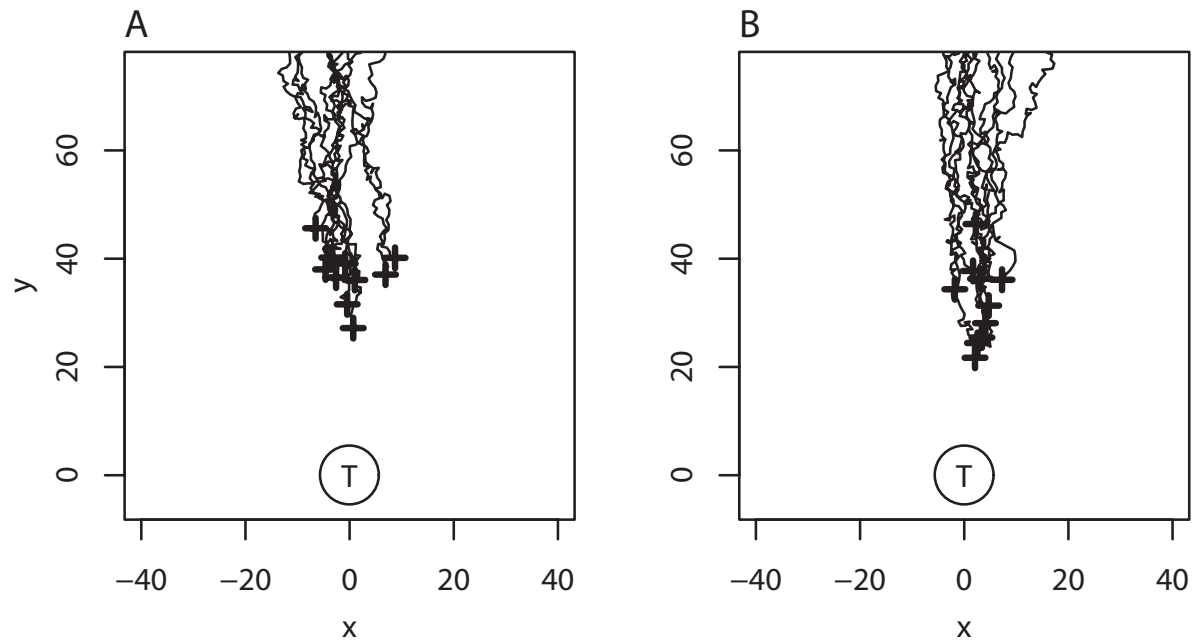

Figure 1: Simulated trajectories for groups navigating toward a target $(T) . A$, Social navigation; $B$, nonsocial navigation. We assume, as we did throughout this research, that groups are aggregated initially. By eye, the difference between the two scenarios is difficult to determine, as initial aggregation and a common target lead to a navigating aggregation in B. See "Methods" for details on the model. Parameter values that are different from the typical values listed in table 1 are as follows: $N=10, r_{\mathrm{I}}=10$, and the simulations are shown over 80 time steps; in $A, \omega=0.5, \sigma_{\mathrm{s}}^{2}=1$, and $\sigma_{\mathrm{o}}^{2}=0.5$; in $B, \omega=1, \sigma_{\mathrm{s}}^{2}=0.7$, and $\sigma_{\mathrm{o}}^{2}=0.1$. For these simulated data, the $x$ and $y$ units have no biological meaning.

thermore, socially navigating groups that actively maintain cohesion are likely to be more resilient to perturbations, such as predatory attacks, and therefore derive more benefits of group membership (Krause and Ruxton 2002).

The individual navigation abilities of animals have been subject to intensive research (e.g., Alerstam et al. 2001; Gould 2004; Simons 2004 and references therein), and influential theoretical studies have suggested mechanisms for the navigation of socially interacting groups (Couzin et al. 2005; Codling et al. 2007; Torney et al. 2010). However, we are not yet able to establish how individuals interact in navigating aggregations. Since many animals move in groups, knowing the mechanisms of group navigation is key when it comes to explaining navigational accuracy, dispersal patterns, population and evolutionary dynamics, and consequently, the design of conservation strategies (Simons 2004). Fundamentally, this is a classic inverse problem, wherein very similar emergent patterns can arise because of quite different processes underlying the behavior at the individual level (see, e.g., Benhamou 2006).

What do we mean by "social interactions" in moving animal groups? Our work builds on the assumption that sociality is expressed by interactions between individual animals that are reflected in the animals' movements relative to one another. We assume that social interactions are different from simple collision-avoidance behaviors. Furthermore, we also consider interactions of individuals with the environment, such as attention to landmarks, to be nonsocial behaviors. Examples of social interactions are the alignment of movement direction between individuals and the tendency of individuals to move toward each other to maintain group cohesion. This loose definition (which we make explicit in our methods) implies that a balance or weighting between the expressions of social and nonsocial behaviors may exist in animals if they are capable of both behaviors. Consequently, we may find not only social or nonsocial groups but also groups of varying degree or relative level of sociality.

Commonly used summary statistics for the group movement of animals, such as the average alignment of individuals, the average distance to the nearest neighbor (Couzin et al. 2002), or the shape of the group (Hemelrijk and Hildenbrandt 2008), do not help to distinguish socially navigating groups from nonsocial groups. For example, efficient individual navigation may lead to accurate alignment of individuals in groups, while simultaneous navigation toward a target and the underlying population density may affect the observed shape and density of the group. Therefore, determining the level of sociality in navigating animal aggregations is an unsolved problem.

Individual-based models have greatly improved our understanding of the movement of social animals (e.g., Reynolds 1987; Couzin et al. 2002, 2005). State-of-the-art models for collectively moving groups of socially interacting individuals are based on the finding that individual actions are the combination of simple behavioral rules that can 
loosely be expressed as "avoid collisions," "align with conspecifics," and "maintain group cohesion." Interactions based on these rules result in complex and realistic movement dynamics (e.g., Couzin et al. 2002; Buhl et al. 2006; Bode et al. 2010). Navigation toward a target or in a target direction can be added to these individual behaviors (Grünbaum 1998; Couzin et al. 2005; Codling et al. 2007).

Here we present a data analysis technique for individual movement trajectories to answer the following question: "given the trajectories of individuals in a group moving toward a known target, to what extent do individuals interact socially?" The basic idea of our approach is that the movement direction of individuals arises from a combination of responses to the environment and to other individuals, and we estimate the relative importance of these responses. We use an individual-based simulation model in which we can adjust the behavioral responses of individuals in a controlled way to demonstrate the robustness and potential of our technique. We then present a case study in which we test our method on shoals of guppies (Poecilia reticulata) moving toward shelter in a tank, and we discuss potential problems and caveats in applications of our technique to empirical data. Our method is generic and can be applied to other scenarios of animal group movement.

\section{Methods}

\section{The "Factor of Sociality"}

Our analysis builds on the work of Eriksson and coauthors, who used a "force-matching" method to determine interaction rules in animal swarms (Eriksson et al. 2010). Unlike in this previous work, for our study we are not interested in the precise nature of the interactions but in the degree to which interactions between individuals are social or nonsocial. At the heart of our analysis lies the assumption that the interactions of each individual, $i$, with their conspecifics and the environment can be described by four interaction types that are inspired by behaviors that are commonly accepted to explain group movement (e.g., Couzin et al. 2002; Krause and Ruxton 2002; Eriksson et al. 2010). We assume that long-range interactions within groups are indicative of social behaviors, whereas shortrange interactions and navigation are nonsocial behaviors. The four interaction types are as follows:

rep $_{i}$ : Short-range interactions that are inspired by collision avoidance (repulsion) and are assumed to be nonsocial.

ori $_{i}$ : Long-range interactions (type 1) that are inspired by alignment with conspecifics (orientation) and are assumed to be social.

att $_{i}$ : Long-range interactions (type 2) that are inspired by attraction to conspecifics to avoid group fragmentation and are assumed to be social.

nav: $_{i}$ : Interaction with the target (navigation; we assume that this is attraction to a fixed-point target) that is assumed to be nonsocial.

Furthermore, we assume that the true direction of motion of each individual at time $t, \mathbf{v}_{i}(t)=\left[\mathbf{x}_{i}(t+T)-\right.$ $\left.\mathbf{x}_{i}(t)\right] /\left|\mathbf{x}_{i}(t+T)-\mathbf{x}_{i}(t)\right|$ (the normalized vector between two consecutive recorded positions $\mathbf{x}_{i}$, for $i, T$ s apart), arises from a linear combination of the above interaction types:

$\mathbf{v}_{i}(t)=\alpha_{1} \mathbf{r e p}_{i}(t)+\alpha_{2} \operatorname{ori}_{i}(t)+\alpha_{3} \operatorname{att}_{i}(t)+\alpha_{4} \operatorname{nav}_{i}(t)$.

The coefficients $\alpha_{k}$ (where $k=1, \ldots, 4$ ) can take positive or negative values. We are estimating the direction of motion of each individual and not the force acting on individuals, as in the study by Eriksson et al. (2010). We do not include an error term in equation (1) because we assume that stochastic effects in the behavior of individuals are not correlated in time and between individuals and that they therefore affect only the variance of the weightings, $\alpha_{k}(k=1, \ldots, 4)$, for the different behaviors. We discuss this assumption in "Results." Also note that in this article we consider movement toward a fixed target point rather than groups moving in a fixed target direction (e.g., Grünbaum 1998; Couzin et al. 2005). It is easy to see that our approach could be extended to include the latter scenario, which is a special case of a "point at infinity" of the more general case we consider here. Recent work on schooling fish suggests that there is no explicit alignment force acting between individuals and that alignment is a result of repulsive and attractive forces (Katz et al. 2011). We do not consider forces between individuals explicitly. As such, the interaction types we include in our analysis could be the result of a combination of more fine-grained interactions that we do not model in detail.

To estimate the contributions of the different types of interactions to the movement of individuals, we estimate the coefficients $\alpha_{k}(k=1, \ldots, 4)$ by minimizing the difference between the right- and left-hand sides of equation (1) across all individuals and instances in time (if we are interested in the group averages) or across all instances in time for one individual (if we are interested in individuals). In practice, we estimate $\mathbf{v}_{i}(t)$ (as seen above) and the values for $\mathbf{r e p}_{i}$, ori $_{i}, \mathbf{a t t}_{i}$, and $\mathbf{n a v}_{i}$ from trajectories for each individual at each time step (or for one individual at each time step). We obtain a vector $\mathbf{y}$ for values of $\mathbf{v}_{i}(t)$ and a matrix $\mathbf{A}$ for the contributions of the different behaviors. Since equation (1) is linear, we have to solve the overdetermined linear system $\mathbf{y}=\mathbf{A w}$, where $\mathbf{w}=$ $\left(\alpha_{1}, \alpha_{2}, \alpha_{3}, \alpha_{4}\right)$, to determine the estimates for the coefficients $\alpha_{k}$ for the different behaviors. This is solved by $\mathbf{w}=\left(\mathbf{A}^{\mathrm{T}} \mathbf{A}\right)^{-1} \mathbf{A}^{\mathrm{T}} \mathbf{y}$ (Eriksson et al. 2010). 
Table 1: Summary of parameters used in the model for group navigation

\begin{tabular}{|c|c|c|c|}
\hline Parameter & Typical value & Range of values & Description \\
\hline$N$ & 40 & $2-140$ & Group size \\
\hline$v_{\mathrm{O}}$ & 1 & & Speed of movement (in units per time step) \\
\hline$\tau$ & 1 & & Time step between movement updates \\
\hline$r_{\mathrm{I}}$ & $(10 N)^{1 / 2}$ & & $\begin{array}{l}\text { Radius of initial random distribution of individuals about start- } \\
\text { ing position (in units) }\end{array}$ \\
\hline$r_{\mathrm{R}}$ & 1 & & Radius of collision avoidance (in units) \\
\hline$r_{\mathrm{O}}$ & 20 & $2-25$ & Radius of alignment interaction (in units) \\
\hline$r_{\mathrm{A}}$ & 30 & & Radius of group cohesion interaction (in units) \\
\hline$\sigma_{\mathrm{s}}^{2}$ & 2 & $0-3$ & $\begin{array}{l}\text { Angular variance of random noise added to navigation compo- } \\
\text { nent of movement (in radians) }\end{array}$ \\
\hline$\sigma_{\mathrm{o}}^{2}$ & .1 & $0-3$ & $\begin{array}{l}\text { Angular variance of random noise added to final orientation (in } \\
\text { radians) }\end{array}$ \\
\hline$\alpha$ & $3 \pi / 2$ & & Viewing angle in sensory zone of individuals (in radians) \\
\hline$\omega$ & .5 & $0-1$ & $\begin{array}{l}\text { Weighting for navigation behavior ( } \omega=0 \text { corresponds to no } \\
\text { navigation) }\end{array}$ \\
\hline
\end{tabular}

From the vector $\mathbf{w}$ we define the "factor of sociality," $\xi=\left(\left|\alpha_{2}\right|+\left|\alpha_{3}\right|\right) /\left(\left|\alpha_{1}\right|+\left|\alpha_{2}\right|+\left|\alpha_{3}\right|+\left|\alpha_{4}\right|\right)$, as the weighting for the social part in the behavior of individuals $(0 \leq \xi \leq 1)$. High values of $\xi$ indicate that social interactions dominate the group dynamics, and low values indicate that nonsocial behaviors are predominant. When we compute $\xi$ for individuals rather than for the average behavior across groups, we denote it as $\xi_{\text {indiv }}$. We consider only the absolute values of the coefficients $\alpha_{k}$ in our computation of $\xi$, despite the fact that they can (and do) take negative values. One way to interpret this is that with the long-range interaction types, we approximate the magnitude of long-range interactions that we assume to be indicative of socially interacting groups but not the precise nature of these interactions. It is likely that the actual interactions in animals are not as simple as suggested by our analysis. However, we show that our approach works in simulations and empirical data. Our long-range interaction types make sense only in groups in which individuals can perceive others. Dispersed populations, in which individuals cannot perceive each other, are not covered by our framework.

To complete the analysis, we must determine how the contributions of the different behaviors are estimated from the data. An important part of our method is approximating from the data the interaction ranges of individuals. This process and further details of the analysis are given in the appendix, available online.

\section{Simulation Framework}

The simulation model presented here does not aim to replicate animal collective motion in all detail. Rather, we suggest a data analysis technique and use representative simulations to demonstrate the robustness and potential of our approach. We simulate groups of $N$ individuals navigating in discrete time and two dimensions toward a fixed target point by extending previous work (Codling et al. 2007). At every time step of length $\tau$, each individual follows adjustable behavioral rules to choose a new direction of movement, and it moves in this direction at a constant speed, $v_{\mathrm{O}}$. We choose $\tau=v_{\mathrm{O}}=1$ (Codling et al. 2007). We consider only groups moving within a homogeneous environment. Individuals start with random initial movement directions from random positions within a circle of radius $r_{\mathrm{I}}$ around the starting coordinate $(0$, $1,000)$ and then navigate toward the origin, $(0,0)$. We use data from the last $100 \tau$ of simulations over $500 \tau$, which is too short for individuals to reach the target but is long enough to avoid recording the initial transitional behavior of groups. To control the level of sociality in our simulated groups, we introduce a weighting, $\omega(0 \leq \omega \leq 1)$, which controls the balance between social and nonsocial behaviors at the individual level (akin to Couzin et al. 2005). Unless otherwise stated all individuals have the same $\omega$ value, but we do explore the case in which a few "informed" individuals guide the rest of the group, which is composed of naive individuals for whom $\omega=0$ (Couzin et al. 2005). In the appendix we give the full details of how we simulated the various behavioral rules and group interactions. Table 1 provides a summary of the model parameters and parameter values used in the simulations.

\section{Results}

Figure 1 illustrates the basic problem that we are considering: it is difficult to distinguish social from nonsocial group navigation. Computing $\xi$ for the two simulations shown in figure 1 allows us to distinguish between the different group behaviors (in fig. $1 A, \xi=0.41$, social nav- 
igation; in fig. $1 B, \xi=0.076$, nonsocial navigation). In this section, we illustrate the robustness of the factor of sociality for varying group sizes and different aspects of individual behavior (e.g., size of individual navigation error) by analyzing our simulated model over a range of parameter values.

\section{Effect of Navigation Weighting, $\omega$}

To illustrate the performance of our approach, we systematically alter the balance between social and individual behaviors in simulations of our model by varying the navigation weighting, $\omega$. In this way, we simulate groups that do not navigate at all $(\omega=0)$, groups in which individuals only navigate and avoid collisions $(\omega=1)$, and groups with intermediate behaviors $(0<\omega<1)$. Figure 2 shows that the factor of sociality, $\xi$, accurately captures these imposed changes in behavior. In figure $2 A \xi$ does not reach its maximum value of $\xi=1$, even if no individual navigation is included $(\omega=0)$. This is explained by the fact that individuals still exhibit nonsocial short-range interactions (e.g., repulsion) in the absence of individual nav- igation. Figure $2 B-2 E$ shows the estimated contribution of the different interaction types. The long-range interaction type 1 (fig. 2C) and navigation (fig. 2E) behaviors are as expected for varying values of $\omega$. As the navigation weighting increases, the contribution of the long-range interaction type 1 decreases and the contribution of navigation increases. The other interaction types are more difficult to interpret (fig. $2 B, 2 D$ ). Individuals in our model always attempt to avoid collisions, irrespective of the value of $\omega$. In sparser groups, however, the group cohesion behavior may dominate over other behaviors. Therefore, both $\alpha_{1}$ and $\alpha_{3}$ are intricately linked to the density of groups, which may explain figure $2 B$ and $2 D$. In general, the results obtained using estimated interaction ranges are very close to those obtained using the real model parameters (see fig. 2 , but see below), which illustrates the robustness of our approach for coarse parameter estimates.

\section{Effect of Group Size}

We tested the effect of group size on the factor of sociality, $\xi$, by keeping fixed all model parameters apart from group
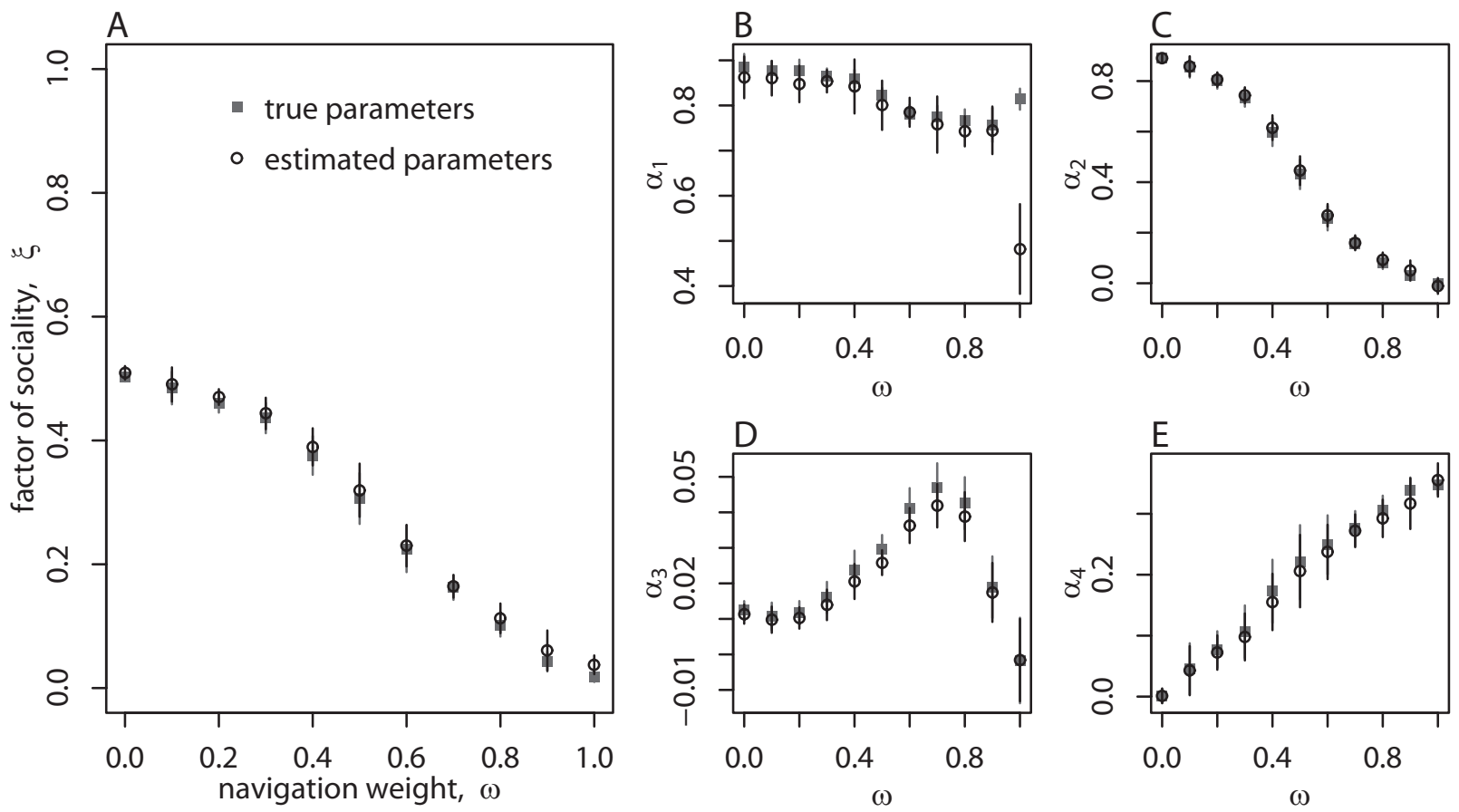

Figure 2: Factor of sociality, $\xi$, and the contribution of different interaction types, $\alpha_{k}$, estimated from simulated trajectories for varying navigation weighting, $\omega$. For comparison, we show the results of the analysis for the true model parameters (gray squares; $r_{\mathrm{R}, \text { est }}=r_{\mathrm{R}}$, $r_{\mathrm{O} \text {, est }}=r_{\mathrm{O}}$, and $r_{\mathrm{A}, \text { est }}=r_{\mathrm{A}}$ ) and for estimated parameters (black circles; $r_{\mathrm{R}, \text { est }}, r_{\mathrm{O} \text {, est }}, r_{\mathrm{A} \text {, est }}$ as described in the appendix, available online). Note that in most cases there is little difference between the true and the estimated parameters, which demonstrates the robustness of the analysis. $A$, As social behaviors are replaced by individual navigation (increasing $\omega$ ), $\xi$ decreases. $B-E$, Estimated coefficients for the contribution of short-range $\left(\alpha_{1}\right)$, long-range type $1\left(\alpha_{2}\right)$, long-range type $2\left(\alpha_{3}\right)$, and navigation $\left(\alpha_{4}\right)$ interactions. See table 1 for parameter values. The average over 10 simulation runs is shown, $\pm 1 \mathrm{SD}$. 
size (see fig. $3 A$ ). While for values of $N$ greater than $30 \xi$ appears to be approximately constant, for smaller values of $N$ it seems to fluctuate. There are a number of possible reasons for this. It may be the result of reduced amounts of data used in the computation of $\xi$. For example, if we use the trajectories of groups of $N$ and $N / 2$ individuals recorded over the same period of time, then we have only half of the data points for our computation for the smaller group. However, figure Al in the appendix, available online, shows that for $N=10$, increasing the length of the trajectories used does not alter the discrepancy in $\xi$ for true and estimated parameters. This indicates that for $N=10$ the length of trajectories used was sufficient, but this may not be the case for smaller values of $N$. We find that the con- tribution of short-range interactions, $\alpha_{1}$, is consistently lower when estimated parameters are used in the analysis. Furthermore, this effect increases with decreasing group size and occurs for values of $N$ of $\sim 20$ or less (see fig. A2 in the appendix). When true parameters are used in the analysis, $\xi$ remains approximately constant for decreasing values of $N$. The reason for this effect can be found in the coarse approximation of the individual interaction ranges in our analysis. In particular, our estimation of the short-range interaction zone of individuals relies on nearest-neighbor distances. For small group sizes, boundary effects create a strong bias, causing estimated nearest-neighbor distances to increase (Cavagna et al. 2008). In our analysis, this results in estimated interaction zones that are larger than the sim-
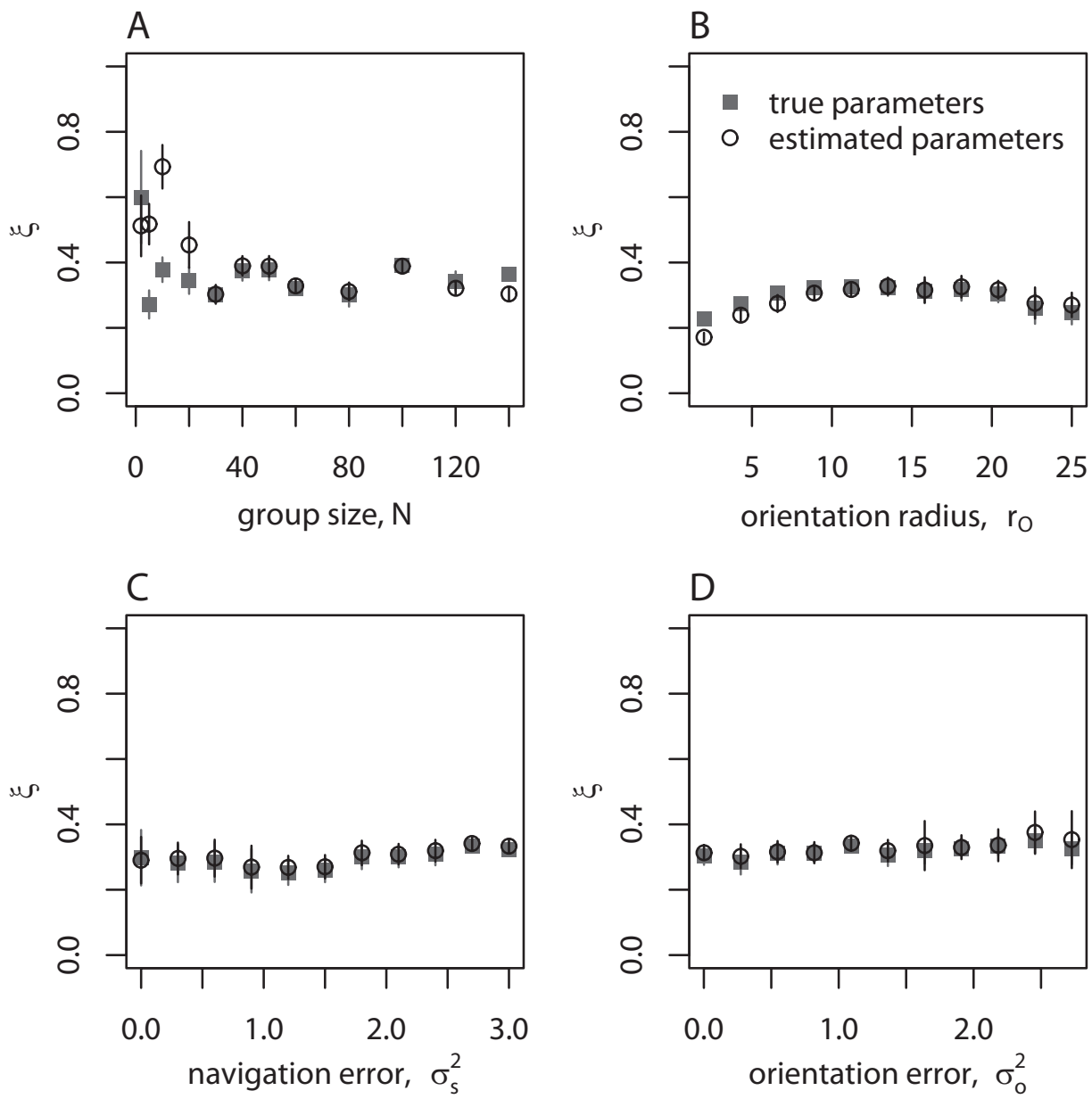

Figure 3: $A$, Factor of sociality, $\xi$, plotted against group size, $N ; B$, radius of orientation interaction, $r_{\mathrm{o}}$; $C$, variance of the navigation error, $\sigma_{\mathrm{s}}^{2} ; D$, variance of the general orientation error, $\sigma_{\mathrm{o}}^{2}$ (e.g., environmental turbulence). For comparison, we show the results of the analysis for the true model parameters (gray squares; $r_{\mathrm{R}, \text { est }}=r_{\mathrm{R}}, r_{\mathrm{O} \text {, est }}=r_{\mathrm{O}}$, and $r_{\mathrm{A} \text {, est }}=r_{\mathrm{A}}$ ) and for estimated parameters (black circles; $r_{\mathrm{R}, \text { est, }} r_{\mathrm{O} \text {, est) }}$ and $r_{\mathrm{A} \text {, est }}$ as described in the appendix, available online). $N=40$ in $B-D$. Note that in most cases there is little difference between the true and the estimated parameters, which demonstrates the robustness of the analysis. See table 1 for parameter values. The average over 10 simulation runs is shown, $\pm 1 \mathrm{SD}$. 
ulated repulsion zones (see fig. A3 in the appendix). Larger estimated interaction zones result in more individuals being wrongly considered in short-range interactions in our analysis, which causes an underestimation of the contribution of short-range interactions. For $N=2$, all of our estimated interaction zones have the same extent, which makes it impossible in our analysis to distinguish between shortrange interactions and type- 2 long-range interactions in a meaningful way. However, figure A4 in the appendix shows that for $N=10$, the qualitative trend in $\xi$ for varying $\omega$ is preserved. Therefore, our coarse parameter estimates can result in quantitative differences, but it is still possible to capture relative differences in $\xi$ between groups of the same size. Furthermore, provided that sufficiently long trajectories are used, the results of our analysis are not affected for $N>5$ if the true simulation parameters are used. For an analysis of the empirical data, on the basis of previous findings or experiments with the study species, it may be useful to consider informed parameter estimates.

\section{Effect of Interaction Radius Size}

In our model, alignment, attraction, and navigation behaviors are averaged in the movement decisions of individuals (see appendix, eqq. [A2] and [A3]). Therefore, changing the size of the behavioral zones might have an impact on the overall weighting of interactions in groups. We tested for such effects by varying the size of the orientation radius, $r_{\mathrm{O}}$, in our simulations (see fig. $3 B$ ). For increasing values of $r_{\mathrm{O}}$, the factor of sociality initially increases, only to decrease again for $r_{\mathrm{O}}>15$ units. This effect reveals aspects of the dynamics of our model. As for how far this could be important for applications to empirical data remains to be seen. Currently, empirical studies have not reached a level of detail sufficient to establish relative sizes of behavioral zones in animal groups. The range of values for the interaction zones we tested include the estimates of Lukeman et al. (2010) that were obtained from empirical data for surf scoters (Melanitta perspicillata). For these results (fig. $3 B$ ) as well as the following (fig. 3C, 3D), the analysis produces almost identical values of $\xi$ for both estimated and true parameters.

\section{Effect of Navigation and Orientation Errors}

Because we did not include noise terms explicitly in our approach (see eq. [1]), it is important to ensure that added noise does not significantly affect the outcome of our analysis. To do this, we varied the magnitude of the noise in individual navigation and that of the overall orientation noise separately (fig. $3 C, 3 D$ ). Other than a slight increase in $\xi$ for increasing noise in both cases, neither of the two types of noise in our model has a pronounced effect on the outcome of our analysis.

Our model assumes noise that is not correlated in time. We expect (but do not test this here) that correlation in the magnitude of noise over time in individual noise terms does not affect our analysis as long as there is no bias in the global direction of the noise. If there was such a noise bias, it would effectively alter the preferred navigation direction of individuals and would be likely to have an impact on our technique.

We disregard individual speeds in our analysis. To ensure that our approach does not work for only one speed, we simulated groups moving at different speeds (see fig. A2 in the appendix). As for the magnitude of the noise, this resulted in only a slight increase in $\xi$ for increasing speeds. Furthermore, the application of our analysis to experimental data in which individuals moved at nonconstant speeds demonstrates the robustness of our approach in this regard.

\section{Varying $\omega$ across Individuals}

So far, we have considered only the case in which all individuals have the same navigation tendency (i.e., all individuals have the same $\omega$ ). That is to say, each individual has the same information about the target (the "many wrongs principle," as described by Simons [2004]). A different and very intuitive alternative mechanism for group navigation is that a small number of "informed" individuals can guide or lead the remainder of the group (Couzin et al. 2005). To explore this scenario, we varied $\omega$ across individuals. For simplicity of illustration, we set $\omega=$ 0.5 for navigating individuals and $\omega=0$ for the remainder of the group. As the fraction of navigating individuals in groups increases, $\xi$ decreases (see fig. A3 in the appendix). This shows that our analysis works on this scenario and that it can distinguish between different levels of sociality in groups, even in the case where not all individuals behave in the same way. Groups with few navigating individuals are therefore navigating socially, according to our method. Calculating $\xi_{\text {indiv }}$, the individual-specific factor of sociality, shows that our method can even distinguish navigating individuals ("leaders") from the rest of the group in simulations (fig. 4; see fig. A4 in the appendix for contributions of interaction types). Although this is an idealized example, it suggests that our method could be used to determine differences in individual behavior and may therefore facilitate answering long-standing questions considering leadership in moving animal groups. 


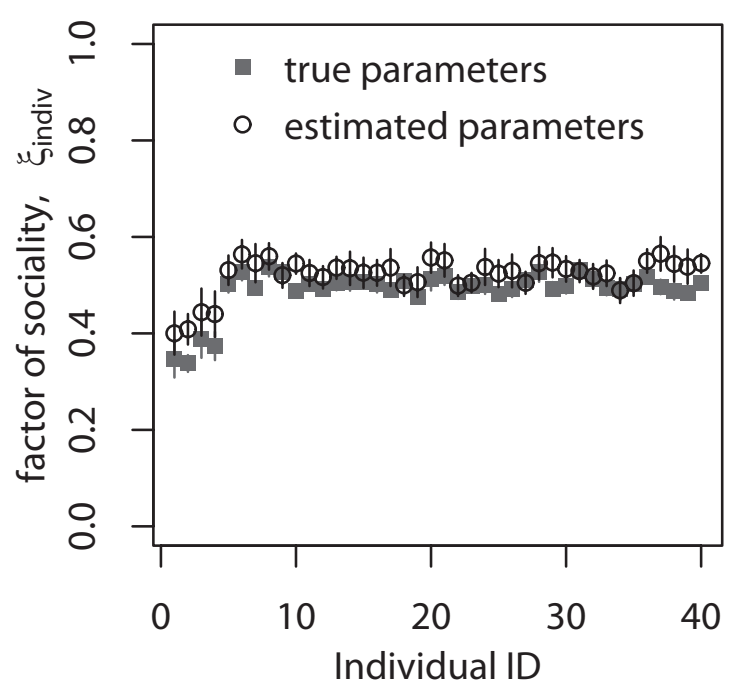

Figure 4: Factor of sociality, $\xi_{\text {indiv }}$, for individuals in simulated groups $(N=40)$. As in figures 2 and 3 , the results for the true model parameters and for estimated parameters are shown. Individuals are assigned unique identities (IDs); individuals $1-4$ are navigating $(\omega=0.5)$, whereas the rest of the group is not $(\omega=0)$. The navigating behavior of individuals $1-4$ is accurately captured in their lower $\xi_{\text {indiv }}$ values. Differences in the results for true and estimated parameters are quantitative but not qualitative. The contribution of different interaction types, $\alpha_{k}$, can be found in the appendix, available online (fig. A7). See table 1 for other parameter values. The average over 20 simulation runs is shown, $\pm 1 \mathrm{SD}$.

\section{Case Study}

We used guppies (Poecilia reticulata) from a captive population to test our technique. Experiments were performed in a white perspex, flat-bottomed square tank with a side length of $60 \mathrm{~cm}$ and a water depth of $6.5 \mathrm{~cm}$. One corner of the tank, equivalent to $\sim 9 \%$ of the tank area, was covered with gravel and shaded by cardboard to provide a shelter and a target for the guppies. The fish were released from a holding cylinder $(10 \mathrm{~cm}$ in diameter) in the corner diagonally opposite from the shelter and filmed from above, using a standard definition camera (Sony Handycam DCR-SX33, 10 frames per second). After fish were released, some groups took longer to start moving. To avoid a possible effect on our analysis of different movement start times, we defined the start of our trials to be when all fish were moving after being released and the end to be when the first fish reached the area covered by gravel. The individual trajectories of the fish were obtained using the open-source tracking software "SwisTrack" (Correll et al. 2006) and previously established methodology (Bode et al. 2010). Because trials did not last for longer than 2:30 min, we could obtain complete trajectories without gaps. A diagram of the experimental setup and an example of individual trajectories can be seen in figure $5 A$.

To establish that guppies showed a preference for the sheltered region, we performed trials with 24 individual male fish. In 21 out of the 24 trials, individuals moved into the sheltered region within $1 \mathrm{~min}$ after being released. However, we do not claim that movement toward the shelter is necessarily representative of guppy behavior in the wild.

For our analysis, we performed trials with single-sex groups of 10 individuals (eight male and six female groups; five trials for each group). The fish could perceive each other across the experimental tank, and we can therefore treat artificial shoals as aggregations and apply our analysis technique to this data. Confinement to the experimental tank restrained the movement of the guppies. Since the experimental setup was identical for all trials and because we did not observe direct interactions with the tank, such as repeated probing of the tank walls, we did not explicitly include interactions with the boundaries in our analysis (but we comment on this possibility later). We assumed the fixed target point to be in the center of the triangular shelter covered with gravel (see fig. $5 A$ ).

Guppies are known to display group fission and fusion dynamics, in which individual fish frequently change group membership (e.g., Croft et al. 2005). This might explain why at times individuals or pairs of fish moved away from the group. To obtain data that were adequate to test our technique, we selected a subset of guppy behavior by using only those trials in which the entire shoal moved in a common direction (not always directly toward the shelter) and eventually reached the shelter. In this way, we selected 14 male and 12 female group trials for our analysis. The mean length of time for which these groups were tracked was $9.6 \pm 1.4 \mathrm{~s}$ (mean \pm standard error).

Because we selected trials, our analysis is not fully representative of natural guppy behavior. Instead, it serves to illustrate our analysis technique for animal trajectories. We did not select shoals of guppies with high factors of sociality, as will become clear in the results.

The fundamental problem of testing our technique empirically is that of finding a suitable control: a priori, we do not know the extent to which individuals interact socially. Our approach is to compare "real groups" of animals, in which individuals can interact, with "artificial groups," in which some or all of the group members cannot interact. If the animals interact, we should find lower values of $\xi$ for artificial groups.

We tested this by performing random permutations of our data. Specifically, we tested the hypothesis that the average factor of sociality across our 26 group trials is not higher than expected if group membership is randomly allocated. Allocating membership of all 260 individuals in 
A

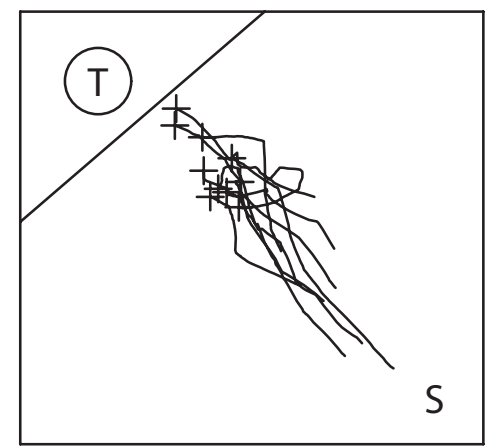

D

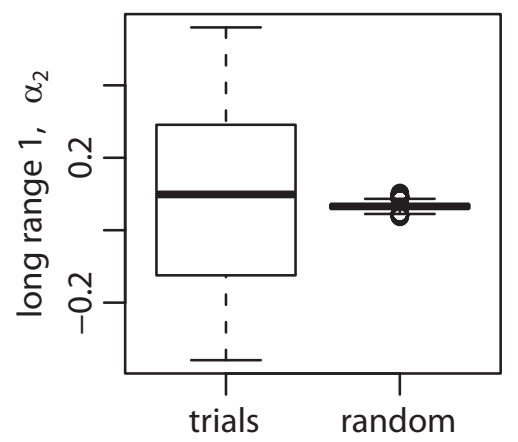

B

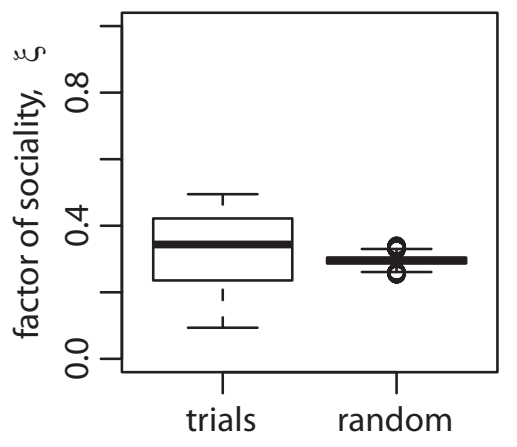

E

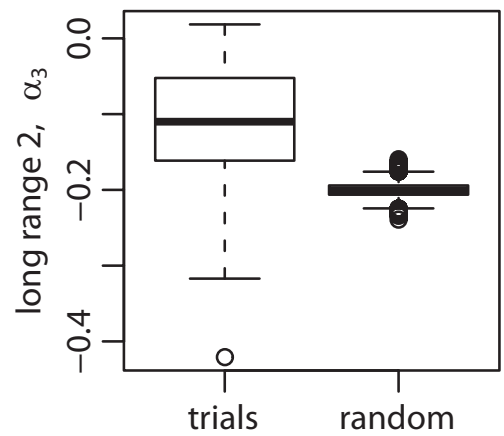

C

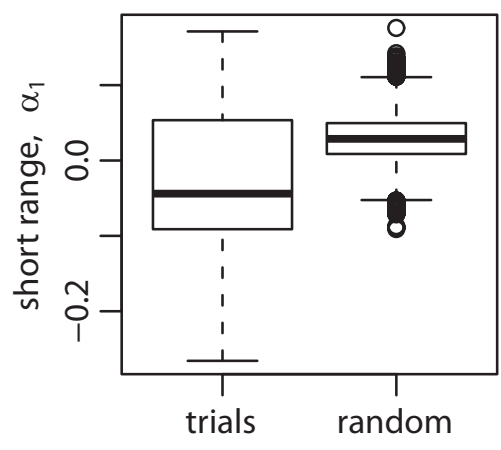

$\mathrm{F}$

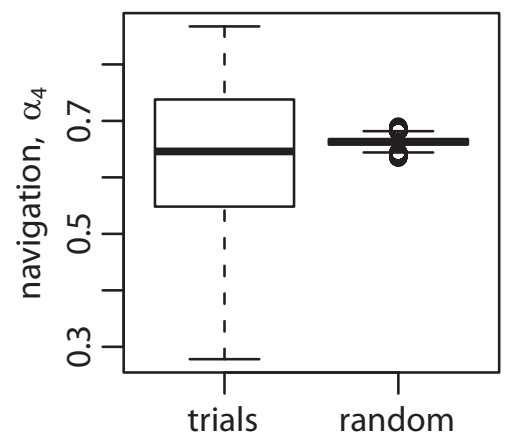

Figure 5: Factor of sociality, $\xi$, and the contribution of different interaction types, $\alpha_{k}$, estimated from the trajectories of real and "artificial" guppy shoals moving toward shelter in a tank. A, The experimental setup, with a release position marked by $S$ and an assumed target position marked by $T$. The triangular shelter region covered by gravel is also indicated. Lines show example trajectories for a shoal of 10 guppies over 1:25 min. Final individual positions are marked with plus signs. We used 26 guppy shoal trials and 10,000 permutations of our data. $B-F$, The factor of sociality and the contributions of the different interaction types. Box plots show medians and twenty-fifth and seventy-fifth percentiles; whiskers indicate 1.5 times the interquartile range, and circles indicate outliers. The distribution of values for the 26 group trials is labeled "trials," and the distribution of the averages obtained for each of the 10,000 permutations of randomly allocated group membership are labeled "random."

our trials at random implies that in most of these permutations of our data, not all individuals in newly created groups come from the same group. Therefore, these individuals do not interact, and we would expect $\xi$ to be lower for these groups than for the group trials.

First, we computed the average factor of sociality across the 26 group trials and found $\xi=0.32 \pm 0.023$ (mean \pm standard error; see also fig. 5). The fact that the minimum and maximum values of $\xi$ for guppy shoals are 0.094 and 0.49 , respectively, demonstrates that we did not select shoals with high $\xi$ values. In one permutation, we randomly allocated the 260 individuals in our group trials to 26 new "artificial groups" of 10 individuals each. Not all group trials lasted for the same length of time. Therefore, we had to combine individual trajectories of different lengths of time in the artificial groups by using all data from the start of each individual track up to the point when the shortest (in time) of the 10 individual tracks in the artificial group ended. We then computed the average of $\xi$ across the 26 artificial groups. We computed the factor of sociality separately for each artificial and each real guppy shoal, using new parameter estimates for each shoal $\left(r_{\mathrm{R}, \text { est }}, r_{\mathrm{O}, \text { est }}, r_{\mathrm{A} \text {, est }}\right.$, see appendix $)$.

In total, we performed 10,000 permutations of our data. Information on the distribution of the averages obtained for the permutations can be found in figure 5 . The fraction of permutations for which the average of $\xi$ across the artificial groups was larger or equal than the average of $\xi$ across our group trials gives an estimate for the probability that $\xi$ is higher for randomly allocated group membership and can be interpreted as a significance value for our hypothesis. We found that only 75 out of 10,000 permuta- 
tions produced higher average values of $\xi$ than the group trials, which corresponds to a significance value of 0.0075 . We therefore reject the hypothesis stated earlier.

This result suggests that our analysis captures differences between the artificial shoals and group trials in the way in which we expect. The average value of $\xi$ is larger for group trials than for artificial groups, and this difference is statistically significant. Because of lack of replication and relatively short trajectories and because our aim was not to specifically investigate guppy behavior, we have not investigated differences in sociality among individual fish.

\section{Discussion}

We have developed an analysis technique that can successfully determine the extent to which navigating animal groups and even individuals in such groups interact socially, given the knowledge of individual trajectories and a fixed target or target direction. Our technique can be applied to movement trajectories of any animal. We present our approach in two spatial dimensions, but it could be extended for an analysis of three-dimensional data. Missing data often result in gaps in animal motion tracking. Because only three consecutive recordings of individual positions are required for each data point, incomplete trajectories could still be used for our analysis (as is also suggested in Eriksson et al. 2010).

Because we intend to illustrate the value of our approach to distinguish between social and nonsocial navigating groups, we refrain from detailed explanations of the dynamics in our simulations. We did not interpret the interaction types in our analysis explicitly as repulsion, alignment, and attraction. The fact that the estimated contribution of the different interaction types can take positive or negative values, even for the simulations (e.g., see fig. $2 D$ ), suggests that the interactions in simulations and empirical data are more complicated than the combination of the four behaviors that inspired our analysis. The important point is that within the parameter range we tested, our approach works qualitatively and gives a relative measure of sociality between groups. Our case study suggests that our assumptions are adequate, despite the fact that they may not capture the actual mechanisms of animal group movement as suggested by Ballerini et al. (2008) or Katz et al. (2011), for example.

In our simulations, we do not consider the possible impact of the environment on the movement dynamics of navigating groups. We model an idealized scenario that may nevertheless be adequate for groups moving in relatively homogeneous environments, such as shoals of fish in the oceans or flocks of birds in the sky. If restraining environmental features or high population densities result in the case where collisions become an important aspect of the dynamics, our approach is unlikely to produce meaningful results. Interactions with obstacles such as tank walls could be included explicitly. Such extensions would require further assumptions or experimentation on behavioral responses of animals and would present a step toward fitting possible behavioral responses to animal movement, as was originally suggested by Eriksson et al. (2010). This provides interesting avenues for further research but is beyond the scope of the work presented here.

For groups that are much larger than the ones we consider $(N>500)$, different properties of moving groups may help to answer our question. For example, recent empirical work has shown that in starling flocks, the average correlation between the movement directions of individuals decreases as a function of the distance between individuals (Cavagna et al. 2010). We suggest that in nonsocial groups, this effect should not be observed.

Some gregarious animals form moving groups that resemble long, narrow streams. Locusts provide an example of this behavior (Buhl et al. 2006), and it has been suggested that it could be explained by pursuit and escape forces in individuals that may be highly anisotropic by focusing on individuals directly behind or in front of others (Romanczuk et al. 2009). If our method is applied to long and narrow marching bands, possibly stretching over kilometers (Buhl et al. 2006), it would certainly fail as a result of the coarse parameter estimates. However, it is possible that our method could be adapted by including blind angles and using informed estimates for the extent of interaction zones. It would certainly be advisable to test adaptations of our method for such scenarios in further simulations.

Recent work has suggested evolutionary scenarios for group navigation. Both Torney et al. (2010) and Guttal and Couzin (2010) suggest that in navigating animal groups, the presence of distinct subgroups of navigating individuals and social followers could be an evolutionarily stable state. This contrasts with mechanisms that assume equal navigation abilities for all individuals in groups (Codling et al. 2007; Faria et al. 2009). Our method can determine the relative weighting given to navigation across individuals in the group, and thereby it presents a unique opportunity to determine which mechanism is occurring across different social animal groups: leadership by a few or an equal navigation investment of all individuals.

It has been suggested that animals alter the strength of their social interactions on the basis of, for example, their individual needs to maintain nutrient levels (Conradt et al. 2009). Our method is ideally suited to assess the impacts of factors such as food deprivation, predation, or social composition of groups on the sociality of groups or of individuals within groups. It may even be possible to quantify the personality of individuals on the basis of sociality 
(Krause et al. 2010). In guppies, for example, individuals have been found to display different and repeatable levels of "boldness," and this may correlate with their social network position (Godin and Dugatkin 1996; Croft et al. 2009). Our method could provide an additional tool with which to quantify these differences between individual: within and across species.

We envisage that our approach could initially be useful to distinguish the level of sociality between different animal species by performing experiments under controllea conditions. In addition, technological advances increasingly make it possible to track animals in the wild (Ballerin et al. 2008; Nagy et al. 2010). Our method requires knowledge of either target coordinates or a target direction, which may not always be available in studies of animal: $\rightarrow$ in the wild. However, there are a number of ways to overcome this problem. While it may not be possible to obtain the trajectories of individuals for the entire duration of their journey, it may nevertheless be possible to determine the endpoint of their journey (e.g., a roost or feeding site), which could be used for the target coordinates. Furthermore, it may be possible to estimate a rough preferred movement direction from the data or other observations given landmarks or reoccurring patterns. As a last resort, the analysis could be repeated over a range of assumed preferred target directions and, on the basis of the assumption that the animals are navigating, the target direction producing the highest contribution of navigation could be used. We suggest that it should therefore be feasible to apply our method to the movement of navigating to understand the mechanisms behind animal navigation in general and ultimately inform conservation or management policies. or even migrating animals in the wild, and this may help $\rightarrow$

depends on topological rather than metric distance: evidence from a field study. Proceedings of the National Academy of Sciences of the USA 105:1232-1237.

Benhamou, S. 2006. Detecting an orientation component in animal paths when the preferred direction is individual dependent. Ecology 87:518-528.

Bode, N. W. F., J. J. Faria, D. W. Franks, J. Krause, and A. J. Wood. 2010. How perceived threat increases synchronization in collectively moving animal groups. Proceedings of the Royal Society B: Biological Sciences 277:3065-3070.

Buhl, J., D. J. T. Sumpter, I. D. Couzin, J. J. Hale, E. Despland, E. R. Miller, and S. J. Simpson. 2006. From disorder to order in marching locusts. Science 312:2317-2326.

Cavagna, A., I. Giardina, A. Orlandi, G. Parisi, and A. Procaccini. 2008. The STARFLAG handbook on collective animal behaviour. 2. Three-dimensional analysis. Animal Behaviour 76:237-248.

Cavagna, A., A. Cimarelli, I. Giardina, G. Parisi, R. Santagati, F. Stefanini, and M. Viale. 2010. Scale-free correlations in starling flocks. Proceedings of the National Academy of Sciences of the USA 107:11865-11870.

Codling, E. A., N. A. Hill, J. W. Pitchford, and S. D. Simpson. 2004. Random walk models for the movement and recruitment of fish larvae. Marine Ecology Progress Series 279:215-224.

Codling, E. A., J. W. Pitchford, and S. D. Simpson. 2007. Group navigation and the "many-wrongs principle" in models of animal movement. Ecology 88:1864-1870.

$\rightarrow$ Conradt, L., J. Krause, I. D. Couzin, and T. J. Roper. 2009. "Leading according to need" in self-organizing groups. American Naturalist 173:304-312.

Correll, N., G. Sempo, Y. Lopez de Meneses, J. Halloy, J. L. Deneubourg, and A. Martinoli. 2006. SwisTrack: a tracking tool for multiunit robotic and biological systems. Pages 2185-2191 in 2006 IEEE/ RSJ International Conference on Intelligent Robots and Systems, Beijing.

$\rightarrow$ Couzin, I. D., J. Krause, R. James, G. D. Ruxton, and N. R. Franks. 2002. Collective memory and spatial sorting in animal groups. Journal of Theoretical Biology 218:1-11.

$\rightarrow$ Couzin, I. D., J. Krause, N. R. Franks, and S. A. Levin. 2005. Effective leadership and decision-making in animal groups on the move. Nature 433:513-516.

$\rightarrow$ Croft, D. P., R. James, A. J. W. Ward, M. S. Botham, D. Mawdsley, and J. Krause. 2005. Assortative interactions and social networks in fish. Oecologia (Berlin) 143:211-219.

\section{Acknowledgments}

N.W.F.B. acknowledges funding by the Natural Environment Research Council. D.W.F. and A.J.W. are funded by Research Councils UK fellowships. We would like to thank three reviewers for informed and constructive comment: that substantially improved this manuscript.

\section{Literature Cited}

$\rightarrow$ Alerstam, T., G. A. Gudmundsson, M. Green, and A. Hedenstrom. 2001. Migration along orthodromic sun compass routes by Arctic birds. Science 291:300-303.

$\rightarrow$ Bajec, I. L., and F. H. Heppner. 2009. Organized flight in birds Animal Behaviour 78:777-789.

$\rightarrow$ Croft, D. P., J. Krause, S. K. Darden, I. W. Ramnarine, J. J. Faria, and R. James. 2009. Behavioural trait assortment in social networks: patterns and implications. Behavioural Ecology and Sociobiology 63:1495-1503.

$\rightarrow$ Eriksson, A., M. N. Jacobi, J. Nyström, and K. Tunstrøm. 2010. Determining interaction rules in animal swarms. Behavioral Ecology 21:1106-1111.

$\rightarrow$ Faria, J. J., E. A. Codling, R. G. Dyer, F. Trillmich, and J. Krause. 2009. Navigation in human crowds; testing the many-wrongs principle. Animal Behaviour 78:587-591.

Godin, J. G., and L. A. Dugatkin. 1996. Female mating preference for bold males in the guppy (Poecilia reticulata). Proceedings of the National Academy of Sciences of the USA 93:10262-10267.

$\rightarrow$ Gould, J. L. 2004. Animal navigation. Current Biology 14:R221-R224.

$\rightarrow$ Grünbaum, D. 1998. Schooling as a strategy for taxis in a noisy environment. Evolutionary Ecology 12:503-522.

$\rightarrow$ Guttal, V., and I. D. Couzin. 2010. Social interactions, information 
use and the evolution of collective migration. Proceedings of thı $\rightarrow$ Nagy, M., Z. Ákos, D. Biro, and T. Vicsek. 2010. Hierarchical group National Academy of Sciences of the USA 107:16172-16177. dynamics in pigeon flocks. Nature 464:890-894.

$\rightarrow$ Hemelrijk, C. K., and H. Hildenbrandt. 2008. Self-organized shap $\rightarrow$ Piyapong, C., J. Krause, B. B. Chapman, I. W. Ramnarine, V. Louca, and frontal density of fish schools. Ethology 114:245-254.

$\rightarrow$ Katz, Y., K. Tunstrøm, C. C. Ioannou, C. Huepe, and I. D. Couzin. 2011. Inferring the structure and dynamics of interactions ir $\rightarrow$ schooling fish. Proceedings of the National Academy of Sciences of the USA 108:18720-18725, doi:10.1073/pnas1107583108. and D. P. Croft. 2010. Sex matters: a social context to boldness in guppies (Poecilia reticulata). Behavioral Ecology 21:3-8.

Krause, J., and G. D. Ruxton. 2002. Living in groups. 1st ed. Oxford University Press, Oxford.

$\rightarrow$ Krause, J., R. James, and D. P. Croft. 2010. Personality in the contex $\rightarrow$ Simons, A. M. 2004. Many wrongs: the advantage of group naviof social networks. Philosophical Transactions of the Royal Society B: Biological Sciences 365:4099-4106.

$\rightarrow$ Lukeman, R., Y. X. Li, and L. Edelstein-Keshet. 2010. Inferring individual rules from collective behaviour. Proceedings of the National Academy of Sciences of the USA 107:12576.

$\rightarrow$ Mellone, U., R. Limnana, E. Mallia, and V. Urios. 2011. Extremely detoured migration in an inexperienced bird: interplay of transport costs and social interactions. Journal of Avian Biology 42:468-472.

Reynolds, C. W. 1987. Flocks, herds and schools: a distributed behavioral model. Computer Graphics 28:25-34.

$\rightarrow$ Romanczuk, P., I. D. Couzin, and L. Schimansky-Geier. 2009. Collective motion due to individual escape and pursuit response. Physical Review Letters 102:010602. gation. Trends in Ecology \& Evolution 19:453-455.

$\rightarrow$ Torney, C., S. A. Levin, and I. D. Couzin. 2010. Specialization and evolutionary branching within migratory populations. Proceedings of the National Academy of Sciences of the USA 107:20394-20399.

Associate Editor: Daniel Roelke Editor: Mark A. McPeek

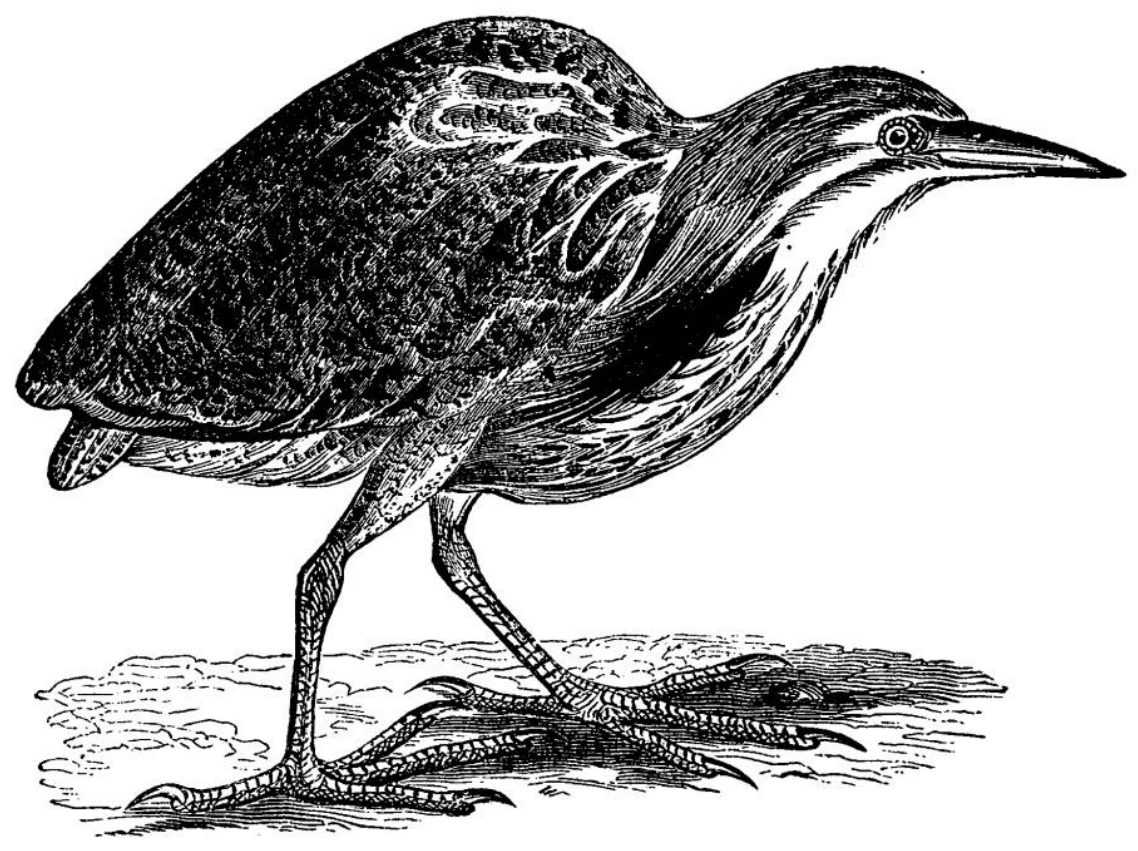

Bittern. "He is a lovely bird in unprejudiced, discriminating eyes; he has no gaudy colors, but his blacks, his brown and yellows, of many shades, all of them pleasing, are so blended as to produce a beautiful, harmonious effect. He loves waste places, for they furnish him safety and food; safety because his enemy, man, is fond of a dry foot; and food, for frogs and snails and snakes and mice, all prime delicacies with our hermit, abound there." From "Bitterns" by William E. Endicott (American Naturalist, 1869, 3:169-179). 